\begin{tabular}{|c|l|}
\hline Title & Formation of curvature singularity al ong vortex line in an axisymmetric, swirling vortex sheet \\
\hline Author(s) & Sakajo, Takashi \\
\hline Citation & $\begin{array}{l}\text { Physics of Fluids, 14(8), 2886-2897 } \\
\text { https://doi.org/10.1063/1.1491255 }\end{array}$ \\
\hline Issue Date & 2002-08 \\
\hline Doc URL & http://hdl.handle.net/2115/5431 \\
\hline Rights & Copyright $\odot 2002$ A merican Institute of Physics \\
\hline Type & article \\
\hline File Information & PF14 8.pdf \\
\hline
\end{tabular}

Instructions for use 


\title{
Formation of curvature singularity along vortex line in an axisymmetric, swirling vortex sheet
}

\author{
Takashi Sakajo ${ }^{\text {a) }}$ \\ Graduate School of Mathematics, Nagoya University, Furo-cho Chikusa-ku, Nagoya, Aichi 464-8602, Japan
}

(Received 12 September 2001; accepted 15 May 2002; published 3 July 2002)

\begin{abstract}
We consider an axisymmetric, swirling vortex sheet in an inviscid and incompressible flow. Caflisch et al. pointed out that the vortex sheet acquired a singularity in finite time, but the property of the singularity was not revealed. In the present paper we show convincing numerical evidences of the singularity formation by applying the same numerical methods as what was used in the study of a two-dimensional (2D) vortex sheet. We find that the radial and axial components of the axisymmetric vortex sheet behave like the $2 \mathrm{D}$ singularity that has been observed in many vortex-sheet motions, while the azimuthal component of the sheet behaves differently. Furthermore, the singularity appears along the vortex line and the first derivative of the vortex sheet strength forms a cusp, while the known singularities are associated with the curvature along curves perpendicular to the vortex lines and the sheet strength has a cusp. (C) 2002 American Institute of Physics. [DOI: 10.1063/1.1491255]
\end{abstract}

\section{INTRODUCTION}

A vortex sheet is a discontinuous surface of velocity field in an inviscid and incompressible flow. It is a simple model that describes an early destabilization process of shear flows at high-Reynolds number.

Singularity formation in vortex-sheet motions is important; mathematically, it serves understanding of the well posedness of the vortex-sheet problems. Physically, it is followed by roll-up of the vortex sheet that results in strong mixing and small-scale production in the flow. In real fluid flows, the singularity would be eliminated by smoothing effects such as viscosity, finite thickness and surface tension, but the influence of the singularity would remain.

A planar vortex sheet is a curve in two-dimensional flow across which the tangential velocity is discontinuous. The curve is represented by a complex-valued function $Z(\Gamma, t)$ $=x(\Gamma, t)+i y(\Gamma, t)$, in which $\Gamma$ is circulation parameter along the sheet and $t$ is time. Moore ${ }^{1,2}$ considered the evolution of the vortex sheet for the following initial condition with a small periodic disturbance:

$$
Z(\Gamma, 0)=\Gamma+i \epsilon \sin \Gamma, \quad-\infty<\Gamma<\infty,
$$

where $\epsilon \ll 1$. He obtained a solution with the following Fourier form:

$$
Z(\Gamma, t)=\Gamma+2 i \sum_{n=1}^{\infty} A_{n}(t) \sin n \Gamma, \quad A_{n}(0)=\frac{\epsilon}{2} \delta_{1 n},
$$

in which the asymptotic expression of $A_{n}(t)$ for large $n$ becomes

$$
\begin{aligned}
A_{n}(t) \sim & t^{-1}(2 \pi)^{-1}(1+i) n^{-5 / 2} \\
& \times \exp \left\{n\left(1+\frac{1}{2} t+\log \frac{1}{4} \epsilon t\right)\right\} .
\end{aligned}
$$

a)Electronic mail: sakajo@math.nagoya-u.ac.jp
The exponential decay in (1) is lost at $t=t_{c}$, where

$$
1+\frac{1}{2} t_{c}+\log t_{c}=\log \left(\frac{4}{\epsilon}\right)
$$

Consequently, since $\left|A_{n}\right| \sim n^{-5 / 2}$ at $t=t_{c}$, the vortex sheet acquires a curvature singularity and behaves like the curve $y=|x|^{3 / 2}$ in the neighborhood of $\Gamma=0$. Subsequent numerical studies by Meiron et al., ${ }^{3}$ Krasny ${ }^{4}$ and Shelley ${ }^{5}$ strongly supported the asymptotic analysis. Then, Caflisch et al. ${ }^{6}$ and Cowley et al. ${ }^{7}$ indicated that the Moore singularity was generic in 2D vortex-sheet motions. Thus the Moore singularity is often referred as "the 2D (curvature) singularity" or "the $\frac{3}{2}$ singularity."

Nie and Baker $^{8}$ and Nitsche ${ }^{9}$ studied singularity formations for axisymmetric vortex sheets. Ishihara and Kaneda ${ }^{10,11}$ and Brady and Pullin ${ }^{12}$ applied Moore's asymptotic analysis to planar 3D vortex sheets for certain perturbations and observed the streamwise curvature singularity. These singularities are associated with the 2D singularity, since they appear along curves normal to the vortex lines in the vortex sheets.

The purpose of our study is to find a singularity formation that differs from the $2 \mathrm{D}$ singularity. We consider the following discontinuous steady swirling flow in the cylindrical coordinates:

$$
\mathbf{u}(r, \theta, z)= \begin{cases}\left(0, \frac{\Gamma_{1}}{2 \pi r}, 0\right) & \text { for } r<R, \\ \left(0, \frac{\Gamma_{2}}{2 \pi r}, 0\right) & \text { for } r>R,\end{cases}
$$

in which $\Gamma_{1}$ and $\Gamma_{2}$ represent the circulations inside and outside the cylinder of radius $R$, respectively. Thus the flow 
defines a cylindrical vortex sheet of radius $R$ and a vortex line of circulation $\Gamma_{1}$ at the center of the cylinder.

Since the cylindrical vortex sheet contains 2D circular vortex sheets, the $2 \mathrm{D}$ curvature singularity certainly appears in finite time. So we assume that the flow is axisymmetric in order to remove the 2D singularity formation. Then the axisymmetric vortex sheet is represented by the cylindrical coordinates $(r(\alpha, t), \theta(\alpha, t), z(\alpha, t))$ of a vortex line on the sheet, where $t$ is time and $\alpha$ is a Lagrangian parameter along the vortex line. The vortex line of circulation $\Gamma_{1}$ on the axis of symmetry never moves due to the symmetry. The vortex sheet evolves according to the following integrodifferential equations (see Caflisch et al. ${ }^{13,14}$ for the details):

$$
\begin{aligned}
\frac{\partial z}{\partial t}= & \frac{\Gamma_{2}-\Gamma_{1}}{2 \pi^{2}} P V \int r^{\prime} \theta_{\alpha}^{\prime}\left(\frac{m}{4 r r^{\prime}}\right)^{3 / 2} \\
& \times\left(\frac{r^{\prime}-r}{1-m} E-\frac{2 r}{m}(E-K)\right) d \alpha^{\prime}, \\
\frac{\partial r}{\partial t}= & \frac{\Gamma_{2}-\Gamma_{1}}{2 \pi^{2}} P V \int r^{\prime} \theta_{\alpha}^{\prime}\left(z-z^{\prime}\right)\left(\frac{m}{4 r r^{\prime}}\right)^{3 / 2} \\
& \times\left(\frac{2}{m}(E-K)+\frac{E}{1-m}\right) d \alpha^{\prime} \\
\frac{\partial \theta}{\partial t}= & \frac{\Gamma_{2}+\Gamma_{1}}{4 \pi r^{2}}
\end{aligned}
$$

in which $r^{\prime}$ and $\theta^{\prime}$ denote $r\left(\alpha^{\prime}\right)$ and $\theta\left(\alpha^{\prime}\right)$ and so on, $E$ $=E(m)$ and $K=K(m)$ are the elliptic integrals of the first kind, and $m$ is given by

$$
m=\frac{4 r r^{\prime}}{\left(z-z^{\prime}\right)^{2}+\left(r+r^{\prime}\right)^{2}} .
$$

Since the integrands in (3) and (4) are singular at $\alpha=\alpha^{\prime}$, they are interpreted in the sense of Cauchy's principle value.

As a matter of fact, the axisymmetric vortex sheet has been already studied by Caflisch et al. ${ }^{13}$ Numerical computation of regularized equations of (3), (4), and (5) showed that a vortex ring formed from the vortex sheet into the outer fluid and the vortex sheet collapsed onto the axis of symmetry. They also pointed out a singularity formation before these nonlinear phenomena, and stated it was related to the $2 \mathrm{D}$ curvature singularity. However, since the 2D singularity formation is eliminated by the axisymmetric assumption, it must be an another singularity formation. We will investigate the nontrivial singularity numerically in the present paper.

We follow the numerical methods that Caflisch et al. ${ }^{13}$ used to compute the evolution of the vortex sheet. Moreover, we adopt the spectral method that Shelley ${ }^{5}$ applied successfully to a $2 \mathrm{D}$ vortex sheet in order to determine the asymptotic form of solution. The numerical methods are briefly explained in Sec. II. In Sec. III, we show numerical evidences indicating that a curvature singularity really forms, and investigate properties of the singularity. Finally, we con- clude the results and discuss the singularity formation in the $3 \mathrm{D}$ cylindrical vortex sheet defined by (2) in the last section.

\section{NUMERICAL METHODS}

We use the same numerical methods as Caflisch et al. ${ }^{13}$ did, in order to compute the evolution of the axisymmetric vortex sheet (3), (4), and (5) except that the calculations are performed in quadruple precision (29 digits). In what follows, we outline the numerical methods. As for the detailed description and analysis of the algorithm, refer to the papers of Refs. 5, 13, 15, and 16 .

We impose a periodic boundary condition on the vortex sheet. That is, denoting $z=\bar{z}+\alpha$, we assume that $r(\alpha, t)$, $\theta(\alpha, t)$, and $\bar{z}(\alpha, t)$ are 1-periodic with respect to $\alpha$. Then, the integral in the right-hand side of (3) or (4) over $\mathbf{R}$, say $I(\alpha)$, is approximated by the following symmetric truncation, $I_{M}(\alpha)$ :

$$
I_{M}(\alpha)=P V \int_{\alpha-(M+1 / 2)}^{\alpha+(M+1 / 2)} f\left(\alpha, \alpha^{\prime}\right) d \alpha^{\prime},
$$

where $f\left(\alpha, \alpha^{\prime}\right)$ symbolizes the singular integrand of (3) or (4), and $\alpha$ runs from -0.5 to 0.5 . As for the truncation period, $M=4$ is sufficient, since the convergence of $I_{M}(\alpha)$ to $I(\alpha)$ is rapid in terms of $M$ as is discussed in Ref. 13 .

We compute the truncated singular integral by an alternate point quadrature; let us discretize $\alpha$-interval $[-0.5,0.5]$ uniformly by even $N$ points. Then, we approximate the integral $I_{M}(\alpha)$ by the summation

$$
I_{M}^{h}(\alpha)=2 h \sum_{k=-\tilde{N}, k: \text { odd }}^{\tilde{N}} f(\alpha, k h),
$$

where $\widetilde{N}=(2 M+1) N+1$ and $h=1 / N$. The quadrature is based on a generalization of the Euler-Maclaurin error expansion for the trapezoidal rule approximation to a singular integral. The first use of the quadrature was reported by Baker. ${ }^{15}$ According to the analysis of the quadrature by Sidi and Israeli, ${ }^{16}$ it attains higher order approximation than the usual trapezoidal rule as long as the integrand $f\left(\alpha, \alpha^{\prime}\right)$ is periodic and sufficiently smooth. It was applied successfully by Shelley ${ }^{5}$ to a $2 \mathrm{D}$ vortex sheet with spectral accuracy. Caflisch et al. ${ }^{13}$ demonstrated that it was also applicable effectively to the computation of the axisymmetric vortex sheet with $O\left(h^{3}\right)$.

The derivative of $\theta$ with respect to $\alpha$, i.e., $\theta_{\alpha}$ in (3) and (4), is obtained by differentiating the discrete Fourier series of $\theta$. The fourth-order Runge-Kutta method is implemented for the temporal integration. In addition, we use the Fourier filtering technique in order to control rapid and spurious growth of the round-off error, since the evolution of the axisymmetric vortex sheet is ill-posed in the sense of Hadamard; ${ }^{13}$ every time step, representing the solution by the discrete Fourier series, we adjust Fourier coefficients that are less than a small preassigned threshold to zero. The technique was used by Krasny ${ }^{4}$ in the numerical computation of 


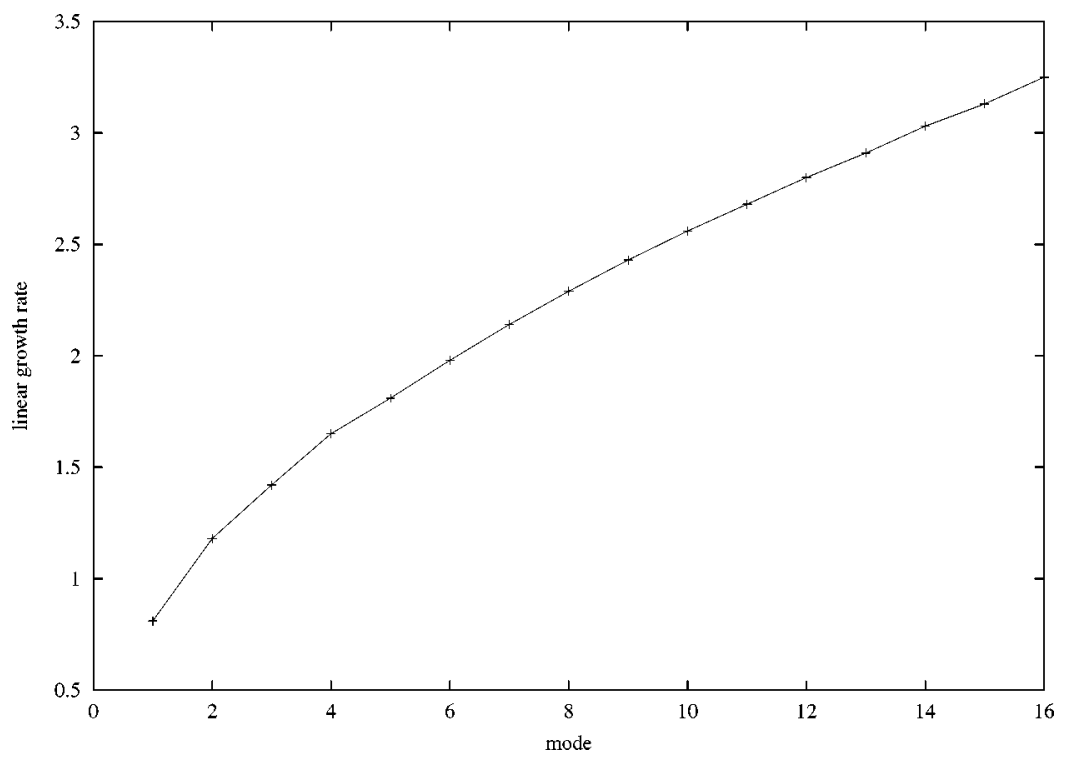

FIG. 1. Linear growth rate of the axisymmetric perturbation $\sigma$ with respect to the mode $k$ for the initial configuration (9). $\Gamma_{1}=6$ and $\Gamma_{2}=0$.

a 2D vortex sheet first and applied effectively to many numerical computations of ill-posed problems. ${ }^{5,8,9,11,13,17} \mathrm{We}$ provide the threshold value with $10^{-25}$, since the computation is performed in quadruple precision. There are two other possible causes of loss of accuracy in the present problem. The first one arises from the stretching of vortex line, which cannot happen in the $2 \mathrm{D}$ case $^{4,5}$ and in the axisymmetric case. ${ }^{8,9}$ The second one occurs when the vortex sheet approaches the axis of symmetry. In fact, Caflisch et al. ${ }^{13} \mathrm{ob}-$ served that the vortex line was elongated greatly and collapsed onto the axis of symmetry after long-time evolution. However, no special procedure to avoid these loss of accuracy is required in the current computation, since the vortex line is slightly deformed and stretched before the singularity time. (See a result in Sec. III A, which shows the overall length of the vortex line is stretched by less than $10 \%$ until the critical time.)

Now, we compare a computational result with a linear stability analysis of the axisymmetric vortex sheet in order to check if the numerical methods described above are correctly implemented to the present problem. Caflisch et al. ${ }^{13}$ showed that the linear growth rate $\sigma$ of the axisymmetric perturbation with the $k$ th mode became approximately

$$
\sigma= \pm \frac{1}{2 \pi}\left(\Gamma_{1}^{2}-\Gamma_{2}^{2}\right)^{1 / 2} \sqrt{|k|}
$$

for large wave number $k$. Hence, when $\Gamma_{1}^{2}>\Gamma_{2}^{2}$, the perturbation is unstable and grows like $\sqrt{|k|}$. Figure 1 shows the linear growth rate of the axisymmetric perturbation as a function of mode $k$ computed by the numerical methods. Initial condition of the vortex sheet and computational parameters are the same as those given in the next section. The growth rate increases like $\sqrt{|k|}$. Hence, the numerical result agrees with the linear stability analysis.

Next, we explain how to estimate the critical time. Assume that, for large $k$, the Fourier coefficients of the solutions are represented asymptotically by

$$
\begin{aligned}
& \left|\hat{r}_{k}(t)\right|=C_{r} k^{-p_{r}} \exp \left(-\delta_{r} k\right), \\
& \left|\hat{z}_{k}(t)\right|=C_{z} k^{-p_{z}} \exp \left(-\delta_{z} k\right), \\
& \left|\hat{\theta}_{k}(t)\right|=C_{\theta} k^{-p_{\theta}} \exp \left(-\delta_{\theta} k\right),
\end{aligned}
$$

where $C_{r}, C_{z}, C_{\theta}, p_{r}, p_{z}, p_{\theta}, \delta_{r}, \delta_{z}$, and $\delta_{\theta}$ are $k$ independent variables, and $\hat{r}_{k}(t), \hat{z}_{k}(t)$, and $\hat{\theta}_{k}(t)$ are defined by

$$
\begin{aligned}
& r(\alpha, t)=1.0+\sum_{k=-N / 2}^{(N / 2)-1} \hat{r}_{k}(t) \exp (2 \pi i k \alpha), \\
& z(\alpha, t)=\alpha+\sum_{k=-N / 2}^{(N / 2)-1} \hat{z}_{k}(t) \exp (2 \pi i k \alpha), \\
& \theta(\alpha, t)=\sum_{k=-N / 2}^{(N / 2)-1} \hat{\theta}_{k}(t) \exp (2 \pi i k \alpha) .
\end{aligned}
$$

At each time step, we compute the time-dependent variables $\delta_{r}(t), \delta_{z}(t)$, and $\delta_{\theta}(t)$ by a form fit of the spectrum to the Ansatz (6), (7), and (8). Then, extrapolating $\delta_{r}(t), \delta_{z}(t)$, and $\delta_{\theta}(t)$, we estimate critical times when they vanish, at which the Fourier coefficients (6), (7), and (8) decay at algebraic orders. The form-fit method to trace singularities was proposed by Sulem, Sulem, and Frisch ${ }^{18}$ for the first time. Krasny ${ }^{4}$ used the least square method for the fit and Richardson extrapolation to estimate the critical time for a $2 \mathrm{D}$ vortex sheet. Shelley ${ }^{5}$ fitted the spectrum to a refined Moore's asymptotic form by requiring that it holds pointwise for consecutive spectral modes and estimated the critical time by a simple extrapolation. In the study of the axisymmetric vortex sheets by Nie and Baker ${ }^{8}$ and Nitsche, ${ }^{9}$ they made use of the pointwise method. We also adopt the Shelley's pointwise method as well. 


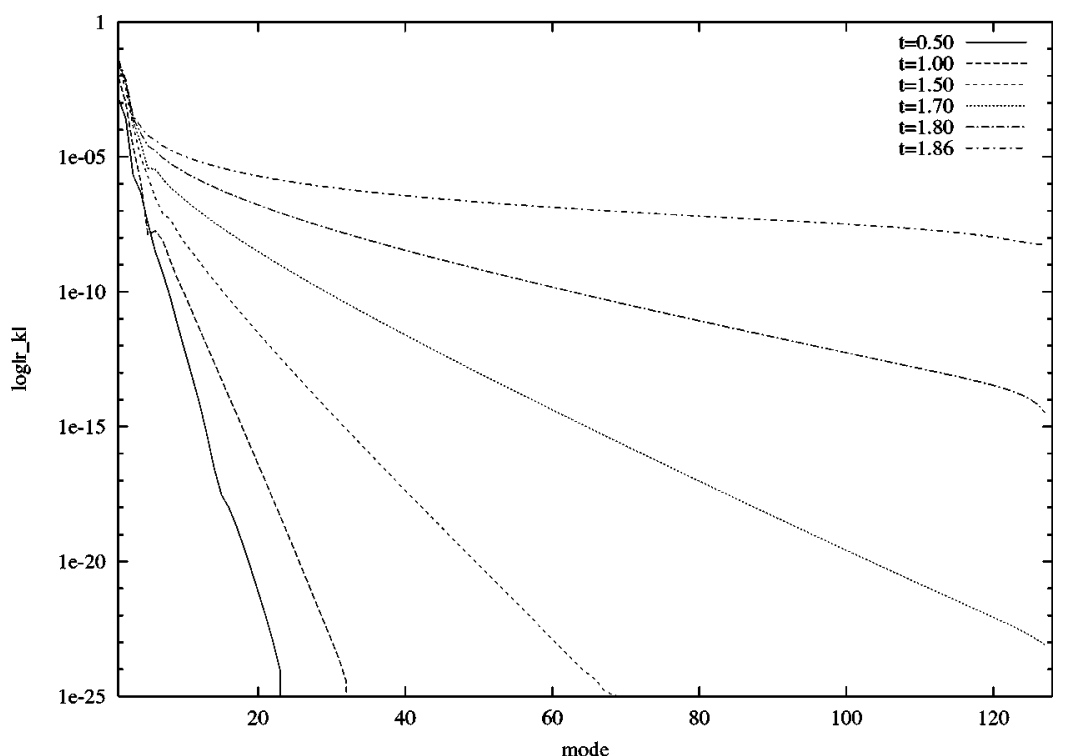

FIG. 2. Discrete Fourier coefficients of the solution $\hat{r}_{k}(t)$ from $t=0.5$ to 1.86 .
Note that since no asymptotic analysis like Moore's is available in this axisymmetric problem, the asymptotic forms (6), (7), and (8) are given just formally. However, the numerical results in the next section indicate that they describe the nascent singularity formation properly.

\section{NUMERICAL RESULTS}

\section{A. Singularity formation in the axisymmetric vortex sheet}

We consider the axisymmetric, swirling vortex sheet of circulation $\Gamma_{1}=6$ inside and $\Gamma_{2}=0$ outside, in which the axisymmetric perturbation is linearly unstable. Initial configuration of the vortex sheet is given by

$$
\begin{aligned}
& r(\alpha, 0)=1.0+\epsilon \cos 2 \pi \alpha, \\
& z(\alpha, 0)=\alpha+\epsilon \cos 2 \pi \alpha, \\
& \theta(\alpha, 0)=\epsilon \cos 2 \pi \alpha .
\end{aligned}
$$

The initial amplitude is $\epsilon=0.01$. We discretize the vortex line by $N=256$ points and the time step size for the RungeKutta method is $\Delta t=0.001$.

Figure 2 shows logarithmic plot of the discrete Fourier coefficient $\hat{r}_{k}(t)$ at $t=0.5,1,1.5,1.7,1.8$, and 1.86 for positive wave numbers. The high wave number spectra grow rapidly at later times.

First, we compute the variable $\delta_{r}(t)$ in the Ansatz (6). Figure 3 shows $\delta_{r}(t)$ versus $k$ at $t=1.65, t=1.7,1.75,1.8$, 1.83, and 1.85. The value of $\delta_{r}(t)$ is independent of wide range of intermediate wave numbers, while it grows largely for higher modes. We regard the $k$-independent value as a proper value for $\delta_{r}(t)$. This is justified by Fig. 4(a), in which we plot $\delta_{r}(t)$ at $t=1.8$ versus $k$ when $N=64,128$, and 256. As $N$ increases, the range of modes where $\delta_{r}(t)$ is uniform expands. Besides, the uniform value has little dependence on $N$. Therefore, as $N \rightarrow \infty$, we rely on the uniform value for $\delta_{r}(t)$ in the Ansatz (6). Back in Fig. 3, the mode-independent

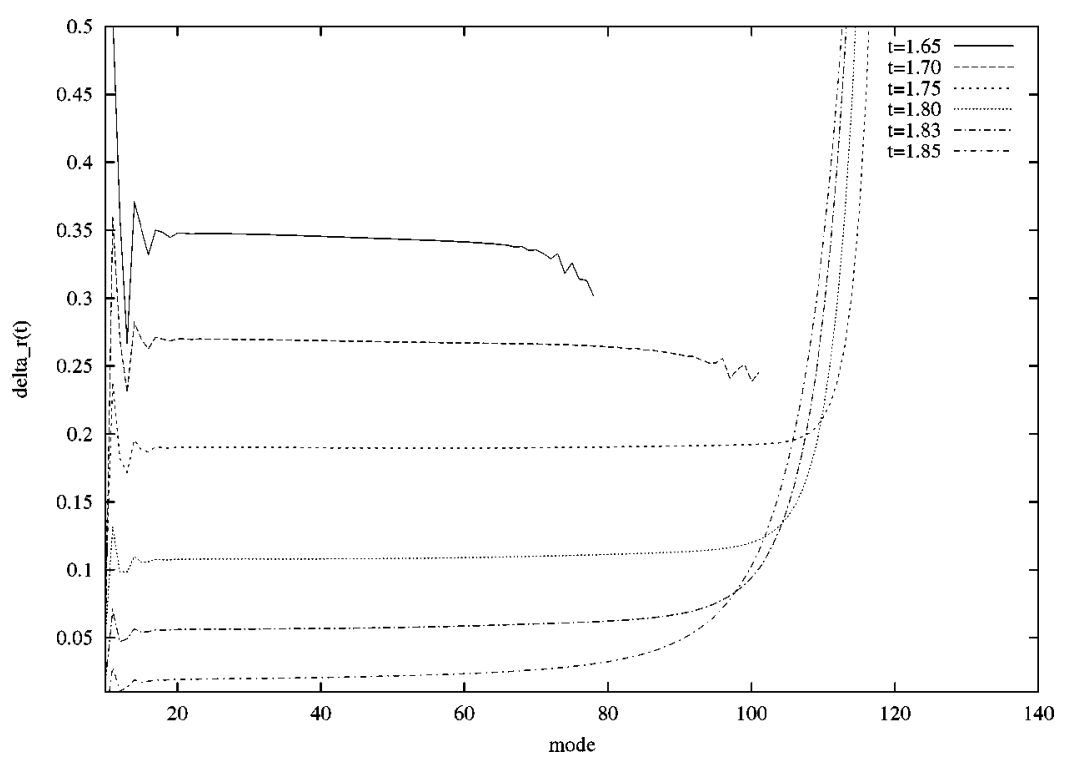

FIG. 3. Plots of $\delta_{r}(t)$ in the Ansatz (6) versus $k$ from $t=1.65$ to 1.85 . They are computed by the pointwise method. 

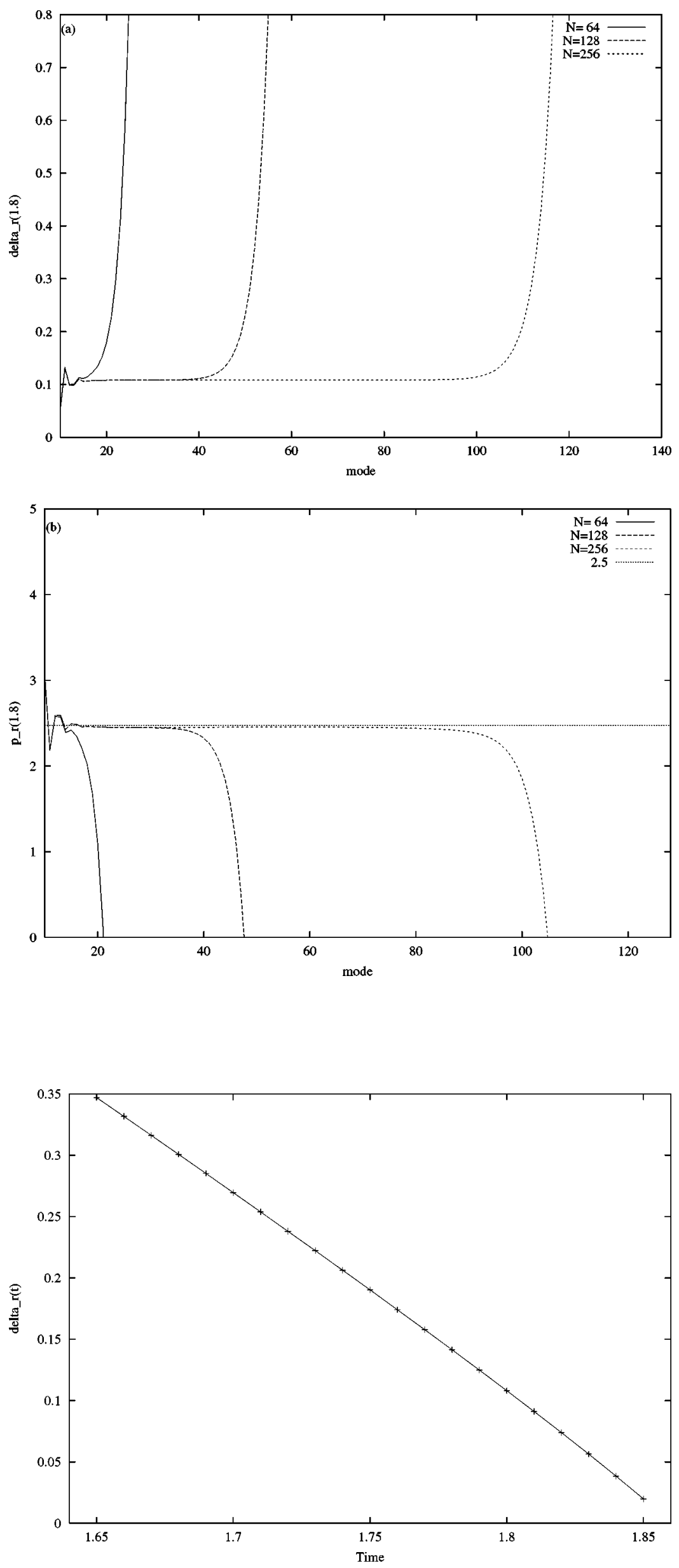

FIG. 4. Plots of (a) $\delta_{r}(t)$ and (b) $p_{r}(t)$ at $t=1.8$ as functions of mode. They are computed by the pointwise method for $N=64,128$, and 256 .

FIG. 5. Plot of $\delta_{r}(t)$ from $t=1.65$ to 1.85 . 


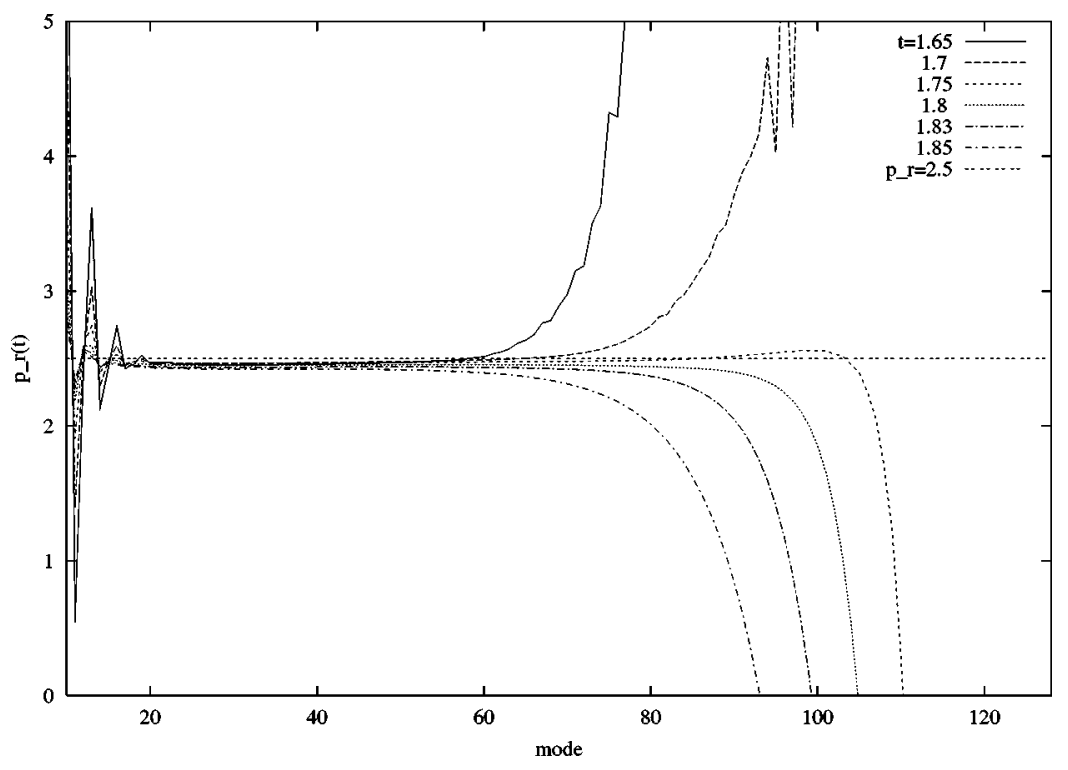

FIG. 6. Plots of index of algebraic decay $p_{r}(t)$ from $t$ $=1.65$ to 1.85 , which are computed by the pointwise method. $\delta_{r}(t)$ tends towards zero as time increases. Figure 5 shows $\delta_{r}(t)$ for $k=30$ from $t=1.65$ to 1.85 . It decreases linearly toward zero. Extrapolating the plot by the least square fit to a linear function, we obtain a singularity time $t^{*}$ such that $\delta_{r}\left(t^{*}\right)=0$, which is $t^{*} \approx 1.864$.

Next, we compute the index of algebraic decay $p_{r}(t)$ in the Ansatz (6). Figure 6 shows $p_{r}(t)$ versus $k$ from $t=1.65$ to 1.85 . They are near 2.5 for intermediate wave numbers independently of time, although they change largely for the higher wave numbers because of the cutoff error. We rely again on the mode-independent value $p_{r}$ as an appropriate value for the index of $k$. This is because, as we see in Fig. 4(b) that shows the value of $p_{r}(t)$ at $t=1.8$ for $N=64,128$, and 256, the range of modes where $p_{r}(t)$ is constant grows as $N$ increases. The index near 2.5 depends on time a little. In fact, it is difficult to insist more than the third digit of $p_{r}$ from the accuracy of the present numerical computations. However, the value of 2.5 is presumable in view of the generic form of the 2D curvature singularity (1).

These observations indicate that the asymptotic form of the discrete Fourier coefficient $\hat{r}_{k}(t)$ right before the singularity time is represented asymptotically by

$$
\left|\hat{r}_{k}(t)\right| \sim k^{-2.5} \exp \left(-\delta_{r}(t) k\right) .
$$

Thus the coefficient behaves like $\left|\hat{r}_{k}\left(t^{*}\right)\right| \sim k^{-2.5}$ at $t=t^{*}$, which suggests that the second derivative of $r$ with respect to $\alpha$ becomes infinite at the critical time. Figures 7(a) and 7(b) are plots of the second derivative and the first derivative of $r$ with respect to $\alpha$ from $t=1.8$ to 1.862 , respectively. The second derivative $r_{\alpha \alpha}(\alpha, t)$ acquires a discontinuity near $\alpha$ $\approx 0.25$ as time approaches the critical time, while the first derivative $r_{\alpha}(\alpha, t)$ is continuous. These figures support the blow-up of the second derivative of $r(\alpha, t)$ at the critical time. On the other hand, because of the asymptotic forms (10), $r$ is represented in the neighborhood of the singular point $\alpha_{0}$ by

$$
r\left(\alpha, t^{*}\right)=1.0+C\left|\alpha-\alpha_{0}\right|^{3 / 2}+\text { less singular term, }
$$

for some constant $C$. Thus the radial component of the vortex sheet behaves like the $\frac{3}{2}$ singularity.

We apply the same numerical procedures successfully to other variables $z(\alpha, t)$ and $\theta(\alpha, t)$. We plot the variables $\delta_{r}(t), \delta_{z}(t)$, and $\delta_{\theta}(t)$ as functions of time in Fig. 8. Every constant decreases linearly to zero. Hence, estimating the critical time of $z$ and $\theta$ by the least square fit, we obtain $t_{z}^{*}$ $\approx 1.863$ and $t_{\theta}^{*} \approx 1.864$. It indicates that $r, z$, and $\theta$ blow up at the same time. Then, we show the indices of $k$ for $z$ and $\theta$, i.e., $p_{z}(t)$ and $p_{\theta}(t)$, at $t=1.75,1.8$, and 1.85 in Fig. 9. While the index $p_{z}$ is near 2.5 for intermediate wave numbers regardless of time, the index $p_{\theta}$ is always larger than 3 and increases slightly as time approaches the critical time. This type of shift for the index was reported by Shelley ${ }^{5}$ in the study of singularity formation in a planar vortex sheet. He observed a transition of the algebraic decay for $y$ component of the vortex sheet from 2.5 to 3 . Anyway, because of the shift it is impossible to claim the second digit of $p_{\theta}$ from the accuracy of present numerical computations. Thus we describe the asymptotic forms of $\hat{z}_{k}(t)$ and $\hat{\theta}_{k}(r)$ as

$$
\begin{aligned}
& \left|\hat{z}_{k}(t)\right| \sim k^{-2.5} \exp \left(-\delta_{z}(t) k\right), \\
& \left|\hat{\theta}_{k}(t)\right| \sim k^{-p_{\theta}} \exp \left(-\delta_{\theta}(t) k\right),
\end{aligned}
$$

for $p_{\theta}>3$. Note that accurate numerical computations with higher resolutions and higher precision floating numbers are required to claim higher digits of the indices $p_{r}, p_{z}$, and $p_{\theta}$ right before the critical time. This is the future problem, since such accurate computations are costly for the time being.

The asymptotic forms (10), (11), and (12) indicate that the second derivative, that is to say, the curvature of the vortex line blows up in finite time. However, it differs from the $2 \mathrm{D}$ curvature singularity. This is because it occurs along the vortex line itself, while the singularities associated with the $2 \mathrm{D}$ curvature singularity ${ }^{1-12}$ appear along curves perpendicular to the vortex lines. Furthermore, it is also different in terms of the vortex sheet strength. According to Caflisch and 

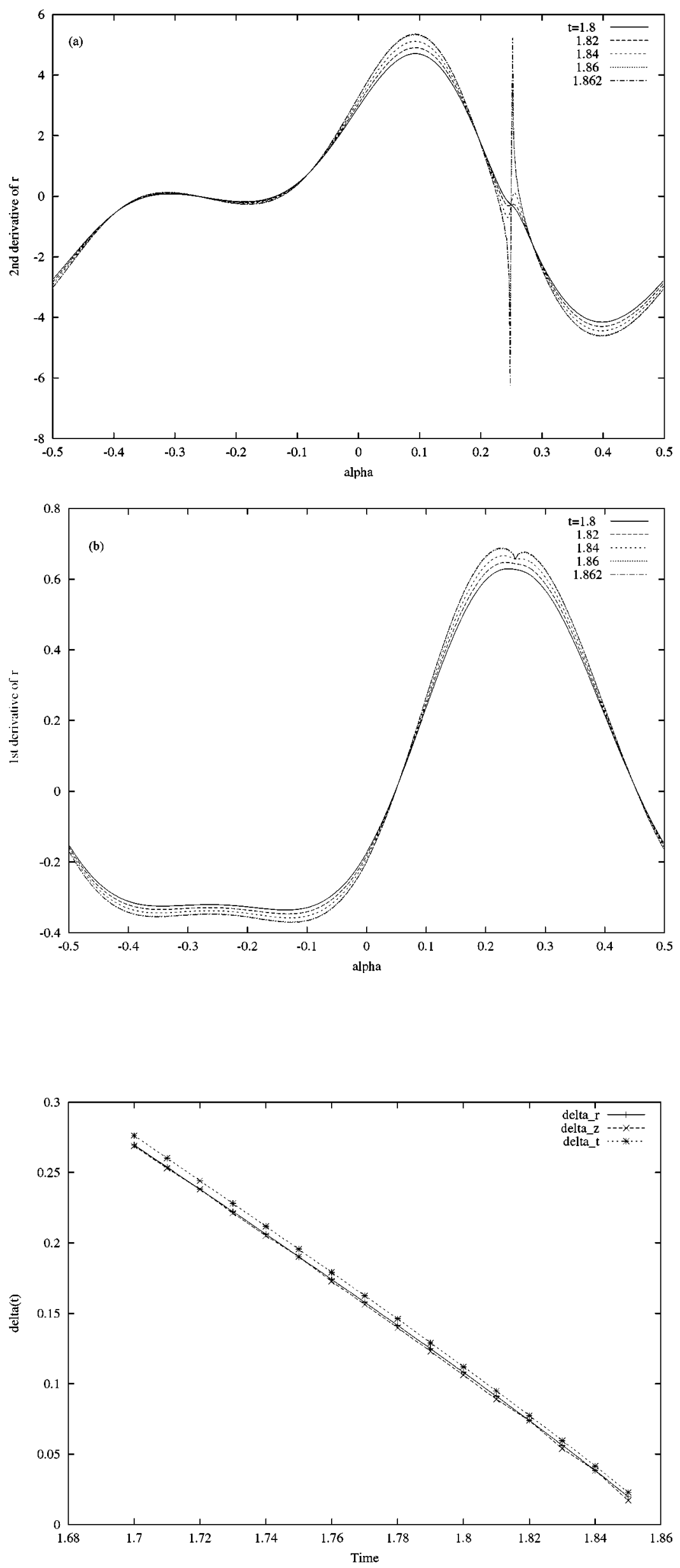

FIG. 7. (a) The second derivative of $r(\alpha, t)$ with respect to $\alpha$ from $t=1.7$ to 1.86 . (b) The first derivative of $r(\alpha, t)$ with respect to $\alpha$ near the singularity time.
FIG. 8. Plots of $\delta_{r}(t), \delta_{z}(t)$, and $\delta_{\theta}(t)$ from $t=1.7$ to 1.85 . 


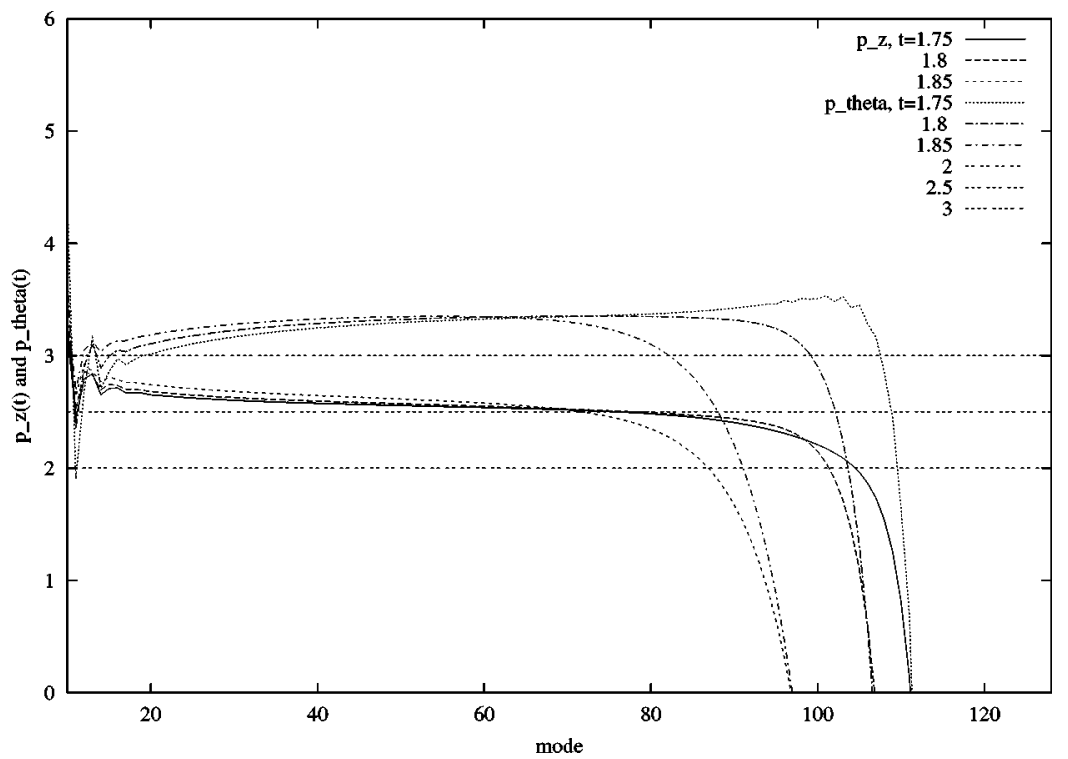

FIG. 9. Plots of indices $p_{z}(t)$ and $p_{\theta}(t)$ from $t=1.7$ to 1.85 , computed by the pointwise method.

$\mathrm{Li}^{14}$ the vortex sheet strength vector in the vortex line, $\kappa(\alpha, t)$, is defined in the cylindrical coordinates by

$$
\kappa(\alpha, t)=\frac{\Gamma_{2}-\Gamma_{1}}{2 \pi r \sqrt{r_{\alpha}^{2}+z_{\alpha}^{2}}}\left(r_{\alpha}, r \theta_{\alpha}, z_{\alpha}\right) .
$$

Therefore, the vortex sheet strength is

$$
|\kappa(\alpha, t)|=\frac{\left|\Gamma_{2}-\Gamma_{1}\right|}{2 \pi r} .
$$

Figure 10 shows the first derivative of the vortex sheet strength $d|\kappa| / d \alpha$ from $t=1.83$ to 1.862 in the neighborhood of the singular point $\alpha_{0} \approx 0.25$. It develops a cusp form at the singular point. Therefore, it is the first derivative of the vortex sheet strength that forms a cusp at the singularity time in the axisymmetric vortex sheet, while the sheet strength develops a cusp in $2 \mathrm{D}$ vortex sheets. This fact plays an impor- tant role in identifying singularity formations in the 3D cylindrical vortex sheet, which is discussed in the last section.

One of the particular features of the present problem is that the vortex line can evolve. Figure 11 shows the stretching rate of vortex line $l(\alpha, t)$, which is defined by

$$
\lambda(\alpha, t)=\frac{\left|\frac{\partial l}{\partial \alpha}(\alpha, t)\right|}{\left|\frac{\partial l}{\partial \alpha}(\alpha, 0)\right|},
$$

from $t=1.7$ to 1.86 . The rate $\lambda(\alpha, t)$ forms a cusp at the point where the singularity occurs as time tends to the critical time, which supports the blow-up of the curvature. The rate is less than one in the neighborhood of the singular point $\alpha_{0}$ and more than one in the other range of $\alpha$. On the other hand, Fig. 12 displays logarithmic plot of the overall length of the vortex line. The vortex line is elongated more than

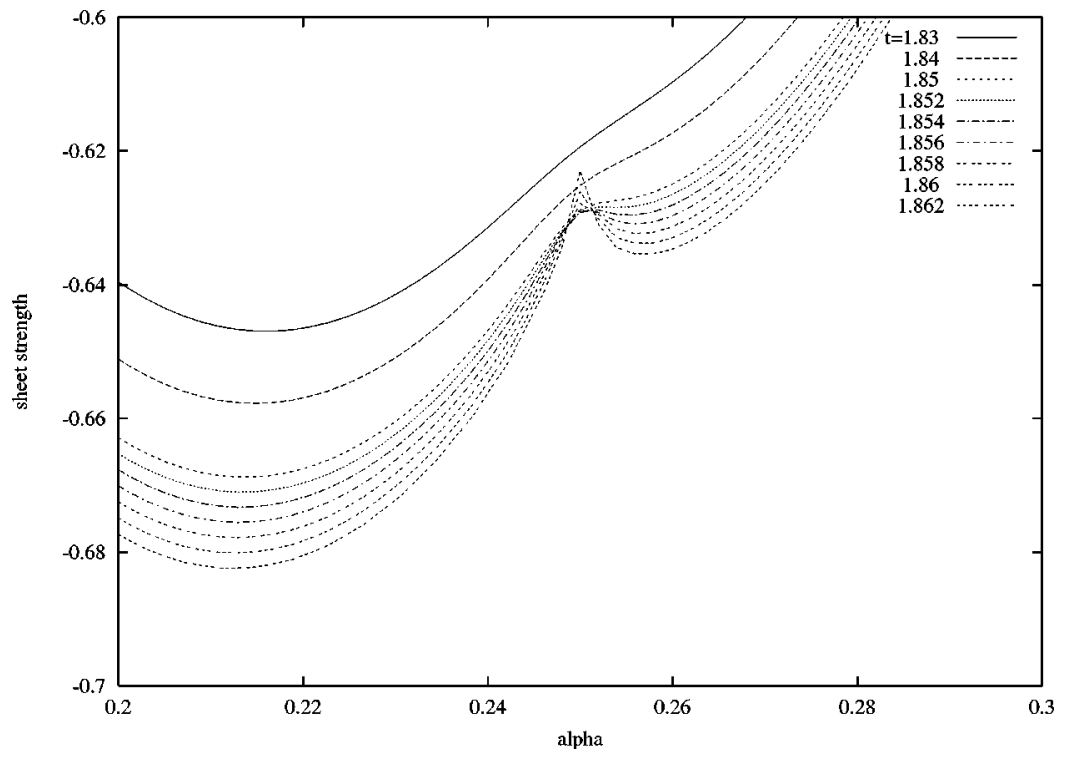

FIG. 10. Plot of the first derivative of the vortex sheet strength $(d|\kappa| / d \alpha)(\alpha, t)$ from $t=1.83$ to 1.862 in the neighborhood of the singular point $\alpha_{0} \approx 0.25$. A cusp forms near the singular point. 


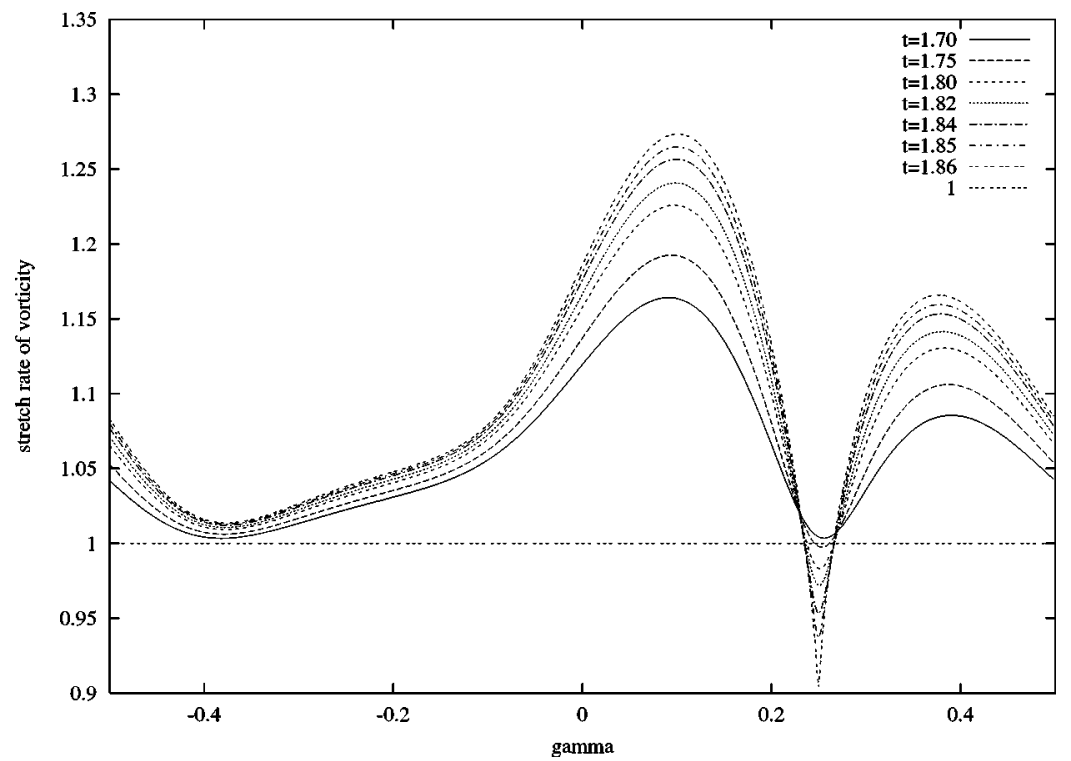

FIG. 11. Plot of the stretching rate of vortex line $\lambda(\alpha, t)$ from $t=1.7$ to 1.86 .

exponentially but bounded. Consequently, the vortex line is locally shortened near the singular point as time tends to the critical time, although the vortex line as a whole is stretched. Thus the singularity formation has little connection with the rapid stretching of the vortex line. It seems to be inconsistent with a conjecture by Beale, Kato, and Majda ${ }^{19}$ claiming that it is the vorticity $|\omega|$ that blows up in an initially smooth 3D Euler flow, if it acquires a singularity. However, it does not contradict to the conjecture because the initial condition of the vorticity in the vortex sheet problem is given by a nonsmooth $\delta$ function, which does not satisfy the assumption of smoothness of the conjecture.

\section{B. Blow-up and amplitude of perturbation}

We study how the singularity formation changes as the initial amplitude $\epsilon$ varies. The same procedure to detect the singularity is applicable to the other value of $\epsilon$. Figure 13(a) shows the critical time $t^{*}$ versus $\log \epsilon$ from $\epsilon=5 \times 10^{-4}$ to
$1.4 \times 10^{-2}$, which indicates that the blow-up time is simply proportional to $\log \epsilon$. The least square fit to these values gives us the relation between $t^{*}$ and $\epsilon$;

$$
t^{*} \approx-0.676 \log \epsilon-1.255 \text {. }
$$

The linear relation is different from what was observed in a $2 \mathrm{D}$ vortex sheet. Indeed, according to the asymptotic analysis by Moore $^{1}$ and the numerical computation by Krasny, ${ }^{4}$ the relation between the singularity time of the vortex sheet, $t_{c}$, and the initial amplitude $\epsilon$ was given by

$$
1+\pi t_{c}+\log \pi t_{c}=-\log 2 \pi \epsilon
$$

for a certain initial condition. This means that the blow-up time $t_{c}$ is proportional to $\log \left(t_{c} \epsilon\right)$. To see the difference clearly, we plot $t^{*}$ versus $\log \left(t^{*} \epsilon\right)$ in Fig. 13(b), which shows that numerical data deviates from a linear relation. This is an another evidence to show the singularity formation in the axisymmetric case differs from that in the $2 \mathrm{D}$ cases.

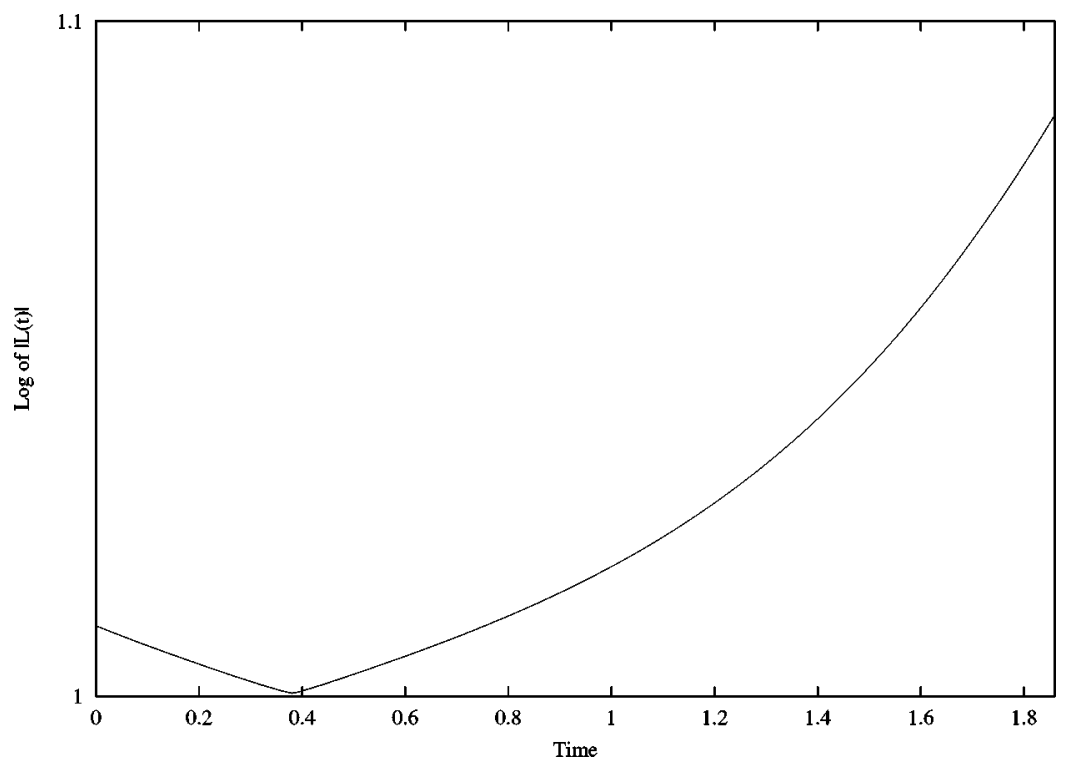

FIG. 12. Logarithmic plot of evolution of the overall length of vortex line. 

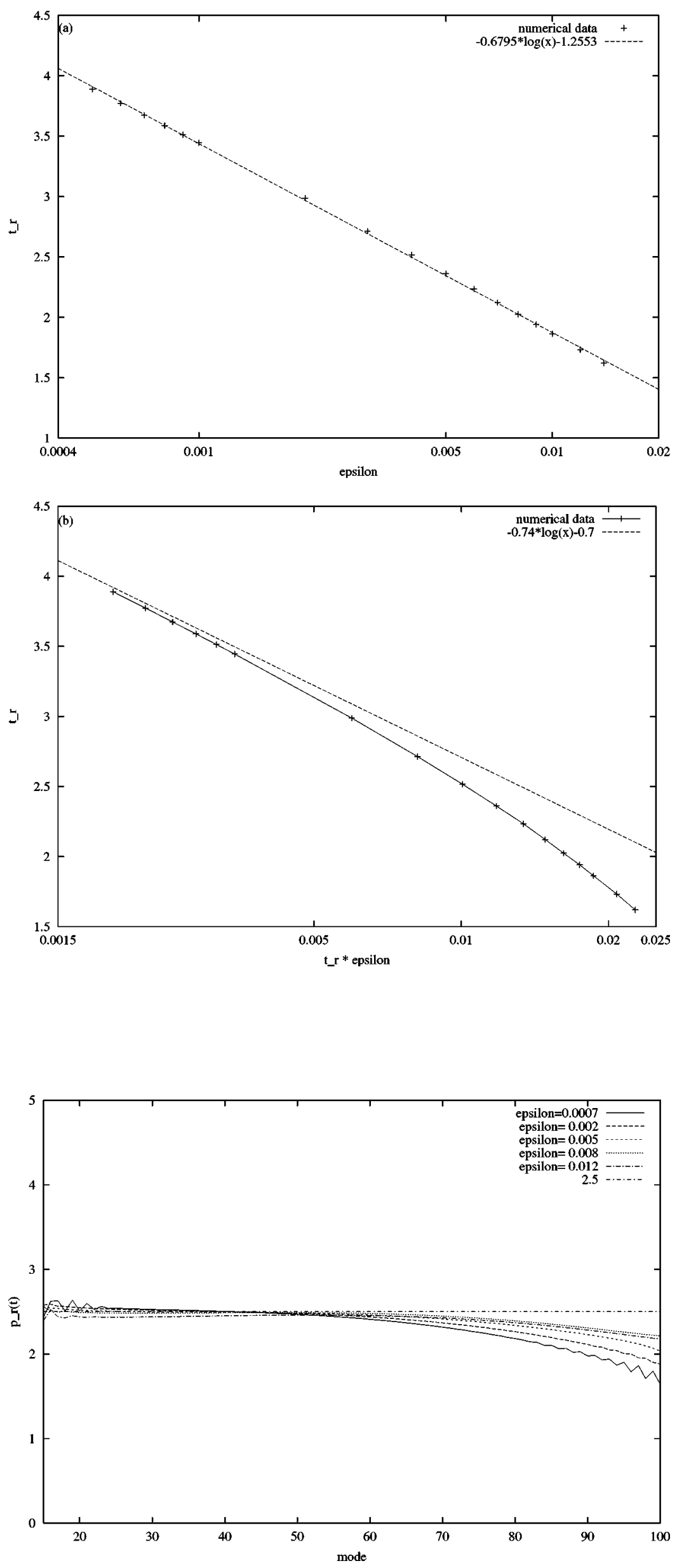

FIG. 13. (a) Estimated blow-up time of $r$ for initial amplitudes of the perturbation $t^{*}(\epsilon)$ versus $\log \epsilon$ from $\epsilon=5 \times 10^{-4}$ to $1.4 \times 10^{-2}$. (b) The blow-up time $t^{*}(\epsilon)$ versus $\log \left(t^{*} \epsilon\right)$.
FIG. 14. Plot of index $p_{r}(t)$ at the time when $\delta_{r}(t)$ $\approx 0.17$ for $\epsilon=7 \times 10^{-4}, 2 \times 10^{-3}, 5 \times 10^{-3}, 8 \times 10^{-3}$, and $1.2 \times 10^{-2}$. 
Figure 14 shows the index of algebraic decay $p_{r}(t)$ at the time when $\delta_{r}(t)$ is about 0.17 for $\epsilon=7 \times 10^{-4}, 2$ $\times 10^{-3}, 5 \times 10^{-3}, 8 \times 10^{-3}$, and $1.2 \times 10^{-2}$. For all $\epsilon$, the value of index is approximately 2.5 for intermediate wave numbers, and it is independent of time. Therefore, the asymptotic form (10) is valid regardless of the amplitude of perturbation as long as it is small.

\section{CONCLUSION AND DISCUSSION}

We verified numerically a singularity formation in an axisymmetric vortex sheet in a swirling flow. We indicated that the second derivative of the vortex line blowed up in finite time and the singularity appeared regardless of the stretching of vortex line at the singular point. The asymptotic formulas of the discrete Fourier coefficients $\hat{r}_{k}(t)$ and $\hat{z}_{k}(t)$ behave like the 2D singularity, while that of $\hat{\theta}_{k}(t)$ is different. The relation between the initial amplitude of the disturbance $\epsilon$ and the blow-up time was also different from the 2D singularity. Furthermore, the present singularity occurred along the vortex line and the first derivative of the vortex sheet strength had a cusp-form in the neighborhood of the singular point. This profile of the singularity is unlike the known singularities, ${ }^{1-5,7-12}$ since they appear along curves normal to the vortex lines and the vortex sheet strength, not its first derivative, forms a cusp at the critical time.

The conclusion suggests that two types of singularities are possible in the 3D cylindrical vortex sheet defined by (2); suppose that a surface $\mathbf{C}(\xi, \eta, t)$ represents the cylindrical vortex sheet, where $\xi$ is the circulation parameter taken along the direction perpendicular to the vortex lines and $\eta$ is a Lagrangian parameter that parametrizes the vortex line. If the surface is assumed to be independent of $\eta$, the motion is equivalent to that of a two-dimensional circular vortex sheet. Then, the 2D streamwise curvature singularity along the circulation parameter, $\max _{\xi}\left|\partial^{2} \mathbf{C} / \partial \xi^{2}\right| \rightarrow \infty$, appears in finite time. The singularity exist continuously in a certain vortex line, where the vortex sheet strength has a cusp profile. On the other hand, if the surface is independent of $\xi$, namely it is axisymmetric, it follows from the present numerical computation that the second derivative along the vortex line, i.e., $\max _{\eta}\left|\partial^{2} \mathbf{C} / \partial \eta^{2}\right|$, becomes infinite at some finite time. In this case, the singular points form a circular curve, where the first derivative of the vortex sheet strength develops a cusp form. In general, for an initial configuration without the symmetries, more complicated phenomenon are expected. Indeed, Sakajo $^{20}$ computed evolution of the 3D cylindrical vortex sheet for a periodic perturbation and observed that generation of a secondary spanwise roll-up structure followed the streamwise roll-up structure due to Kelvin-Helmholtz instability. The primary streamwise roll-up corresponds to the 2D double branched spiral which is considered to be a solution of the vortex-sheet motion after the 2D streamwise singularity formation. ${ }^{21} \mathrm{He}$ also observed the rapid growth of the second derivative of $\mathbf{C}$ along the vortex line just before the secondary roll-up appeared. Actually, since the numerical computation is regularized by the vortex-blob method, the second derivative remains finite throughout the evolution. However, the rapid growth is apparently related to the present axisymmetric singularity formation.

Finally, we discuss the index of algebraic decay of variable $\theta$. It was more than 3 , not $\frac{5}{2}$, although the numerical computations were too inaccurate to resolve the second digit of the index. One of the open problems in singularity formation of 3D vortex sheets is to know if the algebraic decay rate $\frac{5}{2}$ of Fourier modes is generic-like 2D vortex sheets. Shelley ${ }^{5}$ found the $k^{-3}$ decay of the Fourier spectrum in a 2D vortex sheet, but Cowley et al. ${ }^{7}$ and Caflisch et al. ${ }^{6}$ revealed that it was a degenerate case. If the index of algebraic decay is more than three observed in the present study is generic for $3 \mathrm{D}$ vortex sheets, it is a new type of singularity. It is necessary to check if the initial configuration (9) is a degenerate one or not in the future.

\section{ACKNOWLEDGMENTS}

I am very grateful to Professor R. Caflisch and R. Krasny for useful comments and discussion. I completed the work during my stay at the Department of Mathematics of UCLA as a visiting scholar. I also thank the department for support.

${ }^{1}$ D. W. Moore, "The spontaneous appearance of a singularity in the shape of an evolving vortex sheet," Proc. R. Soc. London, Ser. A 365, 105 (1979).

${ }^{2}$ D. W. Moore, "Numerical and analytical aspects of Helmholtz instability," in Theoretical and Applied Mechanics, Proc. XVI IUTAM, edited by F.-I. Niordson and N. Olhoff (North-Holland, Amsterdam, 1984), p. 629.

${ }^{3}$ D. I. Meiron, G. R. Baker, and S. A. Orszag, "Analytical structure of vortex sheet dynamics. Part 1. Kelvin-Helmholtz instability," J. Fluid Mech. 114, 283 (1982).

${ }^{4} \mathrm{R}$. Krasny, "A study of singularity formation in a vortex sheet by the point-vortex approximation," J. Fluid Mech. 167, 292 (1986).

${ }^{5} \mathrm{M}$. J. Shelley, "A study of singularity formation in vortex-sheet motion by a spectrally accurate vortex method," J. Fluid Mech. 244, 493 (1992).

${ }^{6}$ R. E. Caflisch, N. Ercolani, T. Y. Hou, and Y. Landis, "Multi-valued solutions and branch point singularities for nonlinear hyperbolic or elliptic systems," Commun. Pure Appl. Math. 46, 453 (1993).

${ }^{7}$ S. J. Cowley, G. R. Baker, and S. Tanveer, "On the formation of Moore curvature singularities in vortex sheets," J. Fluid Mech. 378, 233 (1999).

${ }^{8} \mathrm{Q}$. Nie and G. Baker, "Application of adaptive quadrature to axisymmetric vortex sheet motion," J. Comput. Phys. 143, 49 (1999).

${ }^{9} \mathrm{M}$. Nitsche, "Singularity formation in a cylindrical and a spherical vortex sheet," J. Comput. Phys. 173, 208 (2001).

${ }^{10} \mathrm{~T}$. Ishihara and Y. Kaneda, "Singularity formation in three-dimensional motion of a vortex sheet," J. Fluid Mech. 300, 339 (1995).

${ }^{11} \mathrm{~T}$. Ishihara and Y. Kaneda, "Singularity formation in the shape of a vortex sheet in three dimensions-numerical simulation," Vortex Flows and Related Numerical Methods II, ESAIM: Proceedings, 1996, Vol. 1, p. 463.

${ }^{12} \mathrm{M}$. Brady and D. I. Pullin, "On singularity formation in three-dimensional vortex sheet evolution," Phys. Fluids 11, 3198 (1999).

${ }^{13}$ R. E. Caflisch, X. Li, and M. J. Shelley, "The collapse of an axisymmetric, swirling vortex sheet," Nonlinearity 6, 843 (1992).

${ }^{14}$ R. E. Caflisch and X. Li, "Lagrangian theory for 3D vortex sheets with axial or helical symmetry," Transp. Theory Stat. Phys. 21, 559 (1992).

${ }^{15}$ G. R. Baker, "Generalized vortex methods for free-surface flows," Waves on Fluid Interfaces (Academic, New York, 1983), p. 53.

${ }^{16} \mathrm{~A}$. Sidi and M. Israeli, "Quadrature methods for periodic singular and weakly singular Fredholm integral equations," J. Sci. Comput. 3, 201 (1988).

${ }^{17}$ J. S. Ely and G. R. Baker, "High-precision calculations of vortex sheet motion,” J. Comput. Phys. 111, 275 (1993). 
${ }^{18}$ C. Sulem, P. L. Sulem, and H. Frisch, "Tracing complex singularities with spectral methods," J. Comput. Phys. 50, 138 (1983).

${ }^{19}$ J. T. Beale, T. Kato, and A. Majda, "Remarks on the breakdown of smooth solutions for the 3D Euler equations," Commun. Math. Phys. 94, 51 (1984).
${ }^{20}$ T. Sakajo, "Numerical computation of a three-dimensional vortex sheet in a swirl flow," Fluid Dyn. Res. 28, 423 (2001).

${ }^{21}$ R. Krasny, "Desingularization of periodic vortex sheet roll-up," J. Comput. Phys. 56, 292 (1986). 